\title{
Predicting the aquatic toxicity of commercial pesticide mixtures
}

\author{
Anja Coors ${ }^{1 *}$ and Tobias Frische ${ }^{2}$
}

\begin{abstract}
Background: Previous studies reported on a large (> 80\%) compliance between the observed toxicity of pesticide mixtures and their toxicity as predicted by the concept of concentration addition (CA). The present study extents these findings to commercially sold and frequently applied pesticide mixtures by investigating whether the aquatic toxicity of 66 herbicidal and 53 fungicidal combination products, i.e., authorized plant protection products that contain two or more active substances, can reliably be predicted by CA.

Results: In more than $50 \%$ of cases, the predicted and observed mixture toxicity deviated by less than factor 2 . An indication for a synergistic interaction was only detected with regard to algal growth inhibition for mixtures of fungicides that inhibit different enzymes of ergosterol biosynthesis. The greatest degree of compliance between prediction and observation was found for the acute toxicity of fungicidal products towards Daphnia and fish, while the greatest degree of underestimation of product toxicity occurred for the acute toxicity of herbicidal products towards Daphnia and fish. Using the lowest available toxicity measures within taxonomic groups as the most conservative approach resulted in a bias towards overestimation of product toxicity, but did not eliminate cases of considerable underestimation of product toxicity.

Conclusions: The results suggest that the CA concept can be applied to predict the aquatic toxicity of commercial pesticide mixtures using the heterogeneous data typically available in a risk assessment context for a number of clearly identified combinations of test species and pesticide types with reasonably small uncertainty.
\end{abstract}

\section{Background}

The environmental risk assessment of plant protection products (PPP) in the European Union (EU) relates to the individual active substances [1]. Depending on the outcome of the EU risk assessment, an active substance (a.s.) may be included in the positive list (the Annex I of the directive 91/414/EEC). Only PPP containing a.s. included in this Annex I can be authorized at the level of the member states. This principle is retained in the new EU regulation 1107/2009 [2], which repeals directive 91/414/EEC and shall apply from June 2011. The new regulation applies not only to PPP and their a.s., but also to other substances contained in commercial PPP, namely safeners, synergists, co-formulants, and adjuvants [2]. The four last component groups are hereafter designated additives. PPP generally represent a mixture of at least one a.s. combined with a number of

\footnotetext{
* Correspondence: a.coors@ect.de

${ }^{1}$ ECT Oekotoxikologie GmbH, Boettgerstr. 2-14, 65439 Flörsheim, Germany Full list of author information is available at the end of the article
}

different formulation additives [3]. The application of a specific PPP does therefore typically result in a potential exposure of non-target organisms to a mixture of chemicals. In addition to formulation additives, PPP can contain two or more active substances. These so-called combination products thereby constitute the specific case of mixtures of pesticidal a.s. that are deliberately released into the environment.

Pesticidal a.s. frequently occur simultaneously in the aquatic environment [4-6]. Consequently, a need has repeatedly been stated to consider the joint effects of pesticide mixtures in the environmental risk assessment [7-9]. Because an experimental testing of all potentially relevant environmental mixtures of pesticides is not feasible simply due to the large number of a.s. and their respective combinations, so-called component-based (in silico) approaches can be considered as an alternative option for a predictive environmental risk assessment that takes joint effects of pesticide mixtures into account. Two basic concepts have been established for predicting additive joint effects based

\section{SpringerOpen ${ }^{\circ}$}


on the known toxicity of the individual mixture components: the concept of concentration addition (CA) for mixtures of substances with similar modes of actions and the concept of independent action (IA, also called response addition) for mixtures of substances with dissimilar modes of action $[7,10,11]$. In addition, combinations of these two concepts for mixtures of dissimilarly and similarly acting substances have been developed $[10,8,12]$. None of these concepts can predict non-additive (synergistic or antagonistic) interactions where the mixture components interfere with each other, e.g., through their toxico-kinetic or toxico-dynamic behavior. A recent state-of-the-art report on mixture toxicity summarizes the scientific background of mixture toxicity concepts as well as the implications of existing approaches for a predictive mixture toxicity assessment in the regulation of chemicals [13].

Deneer [14] and Belden et al. [15] provided an overview on published mixture toxicity studies that explicitly tested the power of one or both of the concepts (CA and IA) to predict the joint toxicity of mixtures of pesticides towards aquatic organisms. Their findings demonstrated that in the majority of experiments ( $80 \%$ and more), mixture toxicity predictions based on CA deviated from the observed mixture toxicity by less than factor 2 . These studies $[14,15]$ thus offer strong evidence that CA is a reasonably reliable concept to predict the mixture toxicity for a range of different a.s. combinations and various single-species endpoints in aquatic toxicology. The limits of these reviews are related to their databases, which consisted solely of experiments that were designed to explicitly test mixture toxicity. The findings obtained with these experiments have a limited generalization potential for prospective risk assessment purposes because the tested pesticide mixtures do not necessarily reflect environmentally relevant pesticide mixtures or mixtures that are present in commercial PPP, i.e., in combination products. Furthermore, the toxicity data for the individual substances and the mixtures in those studies were derived within the same experimental setting, i.e., presumably with identical test species tested in the same laboratory according to identical test protocols. In contrast, the toxicity data typically available for individual a. s. within the context of the regulatory hazard assessment show much higher heterogeneity with regard to test species, test protocols, and measured toxic effects.

By investigating 119 combination products, the present study aims to extend the assessment of compliance between mixture toxicity prediction and observation to a broader range of combinations of pesticides, and specifically to those combinations that are present in commercially applied pesticide mixtures. By using toxicity data from regulatory data bases, the present study explicitly integrates the higher heterogeneity of the data that are typically available to risk assessors and thereby evaluates resulting uncertainty in potential regulatory decisions. As illustrated in Figure 1, the measured aquatic

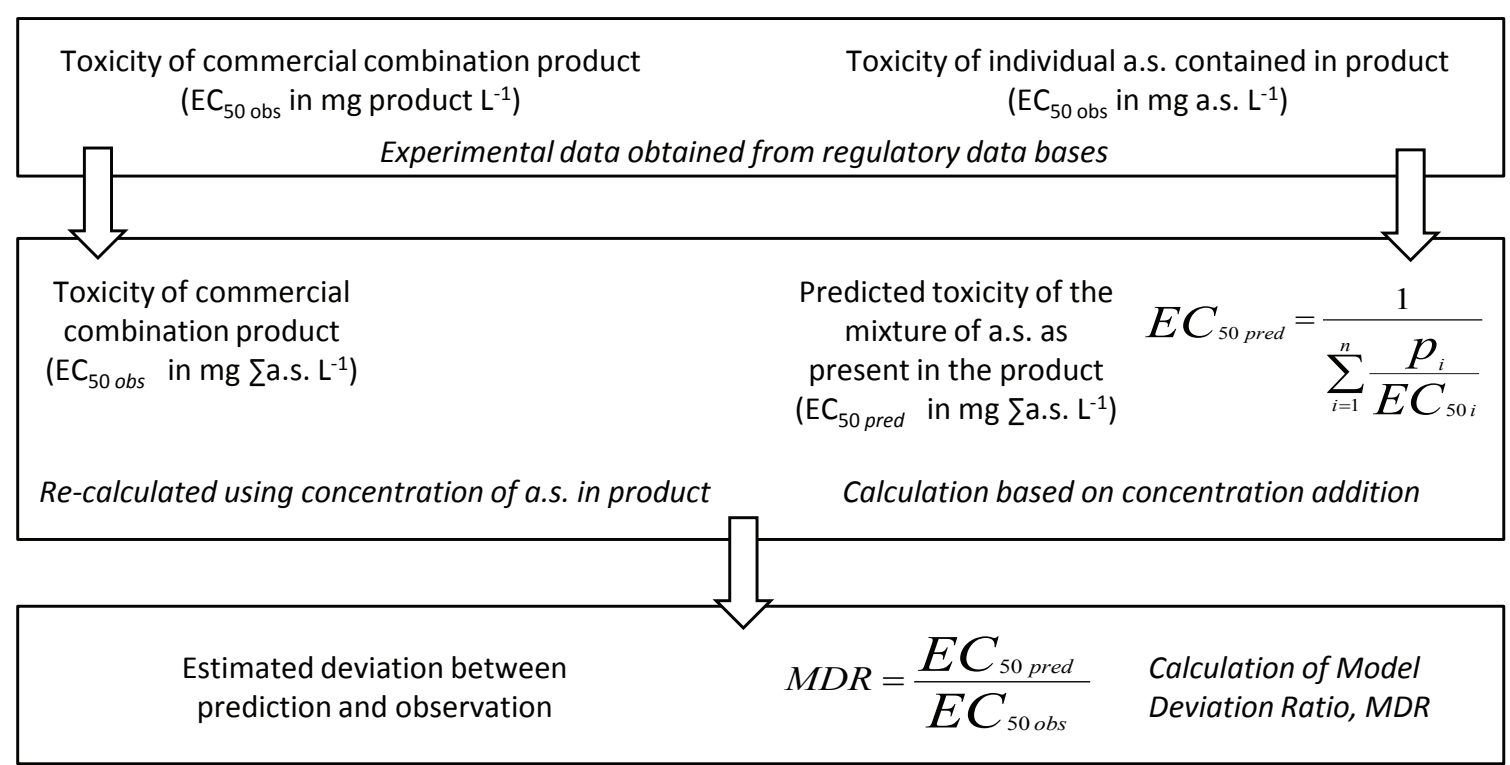

Figure 1 Methodological approach of the present study. Aquatic toxicity data were obtained from regulatory data bases and the toxicity of the mixture was predicted by the concept of concentration addition ( $P_{i}$ denotes the relative proportion of the ith component in the mixture). The predicted toxicity of the mixture was compared to the toxicity of the product, resulting in the model deviation ratio, MDR. The calculations and comparisons were conducted separately within six different endpoints: acute toxicity to aquatic invertebrates and fish as well as growth inhibition of algae and water plants, always using $\mathrm{EC}_{50}$ values; chronic toxicity to Daphnia and fish, using NOEC instead of $\mathrm{EC}_{50}$ values. For detailed information refer to Methods section. 
single-species toxicity of each product was compared with the mixture toxicity prediction calculated according to CA separately for each of six different aquatic standard endpoints used in environmental risk assessment (acute and chronic toxicity to aquatic invertebrates and fish; growth inhibition of algae and water plants). In all cases, only data from the same taxonomic group (i.e., aquatic invertebrates, water plants, algae or fish) were combined for prediction and comparison. Likewise, chronic and acute endpoints were never combined. The predictions for the products were derived separately based on two different data sets of toxicity data of the individual a.s. The first data set was collected from the database of the German Federal Environment Agency and consisted of toxicity data that were selected on the basis that the toxicity test of the individual a.s. was in terms of test species, exposure conditions, and measured toxic effect as similar as possible to the available test conducted with the product in which this a.s. was contained. The second data set was collected from the socalled "list of endpoints" of the EU authorization procedure for pesticide a.s. During this procedure, the lowest toxicity measure within a taxonomic group and endpoint (e.g., algal growth inhibition or toxicity to aquatic invertebrates) is selected by the regulatory authorities involved in the review process based on all available validated toxicity data. These two data sets are referred to as "most similar endpoints" and "most sensitive endpoints" in the following.

This study aims at two objectives: (1) To explore the suitability of a CA-based mixture toxicity prediction as a substitute for the toxicity testing of combination products, i.e., a component-based approach instead of a whole-mixture testing approach. (2) To obtain information about the uncertainty that is related to a CA-based mixture toxicity prediction for commercial pesticide mixtures. Particularly, the assessment based on the second data set ("most sensitive endpoints") integrates the data heterogeneity that has to be taken into account when the toxicity data of the individual a.s. derived in the typical regulatory assessment procedure are used for the mixture toxicity prediction. In addition, the assessment with this data set is expected to be more conservative, because the lowest toxicity measures (median effect (lethal) concentration, $\mathrm{E}(\mathrm{L}) \mathrm{C}_{50}$, and no-observed-effectconcentration, NOEC) within each of the six endpoints are used for the prediction. The frequency distribution of the model deviation ratio (MDR), which had been introduced by [15], was used as illustration of the uncertainty of the CA prediction. The larger the percentage of cases where the observed product toxicity deviates from the prediction, the higher the degree of uncertainty in decisions based on such a mixture toxicity prediction. MDR values greater than 1 indicate that the CA prediction underestimates the toxicity of the mixture, i. e., the combination product is more toxic than predicted. MDR values smaller than 1 indicate that the CA prediction overestimates the toxicity of the mixture, i.e., the combination product is less toxic than predicted. Particularly the frequency of underestimating the toxicity of a PPP is seen as a proxy for the degree of protectiveness of a CA-based environmental risk assessment and will be the main focus of the present study.

\section{Results}

\section{Composition of combination products authorized in Germany}

There were 247 combination products (23.6\%) among the 1046 PPP authorized in Germany on August 4, 2008. The vast majority of these products contained two a.s. $(84 \%)$, while $13 \%$ contained three and $3.1 \%$ contained four a.s., 161 of the 247 combination products were originally authorized products, while 86 products represent subsequent authorizations under a different trade name. Excluding the 26 combination products that are used for seed treatment, the remaining 135 originally authorized combination products belong mainly to two groups: 69 products containing only herbicidal a.s. and 58 products containing only fungicidal a.s. The remaining eight combination products contain insecticides, pheromones, or acaricides, but in no case herbicidal or fungicidal active substances. Table 1 provides an overview on the a.s. composition of the herbicidal and fungicidal combination products with regard to their pesticidal mode of action according to HRAC and FRAC $[16,17]$. Products that contain a.s. with a similar mode of action were clearly underrepresented, while the diversity among products with dissimilarly acting a.s. was very high given that already 34 products represented a unique mode-of-action combination.

\section{Comparing predicted and observed aquatic toxicity of combination products based on "most similar endpoints"} For 66 of the 69 herbicidal and for 53 of the 58 fungicidal combination products, aquatic single-species toxicity data were available in the database of the German Federal Environment Agency (UBA) for the formulated product and all respective a.s. For a number of products and a.s., $\mathrm{EC}_{50}$ or $\mathrm{NOEC}$ values for some endpoints were not available as exact numerical values but only as censored data (i.e., $\mathrm{EC}_{50}$ or NOEC as greater than the highest tested concentration or smaller than the lowest tested concentration). Consequently, the MDR could not be calculated as a numerical value in 100 of in the total 441 cases where a comparison of predicted and observed toxicity of the product was in principle possible. Censored or not-determinable MDR values were more frequently obtained among the herbicidal products 
Table 1 Composition of herbicidal and fungicidal combination products

\begin{tabular}{lll}
\hline & Herbicides & Fungicides \\
\hline Number of combination products (total) & 69 & 58 \\
$\quad$ - with similar MoA & 26 & 6 \\
$\quad$ - with dissimilar MoA & 43 & 52 \\
Number of different a.s. & 60 & 60 \\
Number of represented MoA groups & 14 & 17 \\
Similar MoA combinations (number of products) & $\mathrm{O}(10), \mathrm{B}(11), \mathrm{C}(4), \mathrm{F} 1$ (1) & $\mathrm{G} 1(6)$ \\
Two most frequent dissimilar MoA combinations (number of products) & $\mathrm{O}$ and C (5), & $\mathrm{G} 1$ and C3 (9), \\
Number of products with unique MoA combination & $\mathrm{O}$ and B (4) & 17 \\
Number of combination products here assessed & 17 & 53
\end{tabular}

Given is the number of originally authorized combination products that contain a.s. with a similar or a dissimilar pesticidal mode of action (MoA), respectively. In addition, the number of different a.s. and MoA groups represented in the combination products is indicated together with the MoA combination most frequently represented and the number of products with a MoA combination encountered only once. Classification of pesticidal mode of action was done according to HRAC [16] and FRAC [17]: HRC group O, synthetic auxin; HRAC group B, inhibitor of branched chain amino acid synthesis (ALS inhibitor); HRAC group C, inhibitor of photosystem II; HRAC group F1, inhibitor of carotenoid synthesis; FRAC group G1, inhibitor of C14-demethylase in ergosterol biosynthesis (DMI fungicide); FRAC group C3, inhibitor of cytochrome $c$ oxidoreductase at complex III in the mitochondrial electron transport; FRAC group G2, inhibitor of $\Delta^{14}$-reductase and $\Delta^{8}$ to $\Delta^{7}$-isomerase in ergosterol biosynthesis.

(75 of 255 comparisons, 29.4\%) than among the fungicidal products $(13.4 \%)$. Acute toxicity towards Daphnia and fish recorded with 81 cases is the majority of censored data. This chapter focuses first on the non-censored MDR values, while the censored MDR values are taken into account in subsequent chapters.

Figure 2 shows the frequency distribution of the 274 non-censored MDR values that were calculated using the $\mathrm{EC}_{50}$ estimates available for four of the six endpoints (aquatic invertebrate and fish acute toxicity as well as algal and Lemna growth inhibition), i.e., excluding MDR values based on NOEC values. A deviation of less than factor 2 between prediction and observation (MDR between 0.5 and 2) was clearly the most frequent result (53.6\%), while the frequency of more extreme MDR values decreased considerably to both sides. The MDR values ranged from 0.0016 to 794.8 , i.e., from about a 600 -fold overestimation to an almost 800 -fold underestimation of product toxicity. The median of these 274 MDR values was determined as 0.99 , which indicates a highly symmetric distribution. Hence, the product toxicity was as frequently underestimated by the CA concept as it was overestimated. There was no significant difference between products composed of a.s. with a similar (median of MDR values, $1.06 ; n=62$ ) and a dissimilar (median of MDR values, $0.98 ; n=212$ ) pesticidal mode of action (Mood's median test, $p=0.319$ ). The median MDR value of herbicides $(0.845, n=140)$ and the median MDR value of fungicides $(1.175, n=134$ ) differed significantly (Mood's median test, $p=0.008$ ). Averaged over all endpoints, the aquatic toxicity of herbicidal combination products tended to be more frequently overestimated and that of fungicidal combination products more frequently underestimated by CA. Because of this significant difference in their median MDR values, herbicidal and fungicidal combination products are analyzed in the following separately for the six investigated aquatic endpoints.

\section{Herbicidal combination products}

The frequency distribution of the non-censored MDR values calculated for the herbicidal combination products is shown in Figure 3 for each of the six

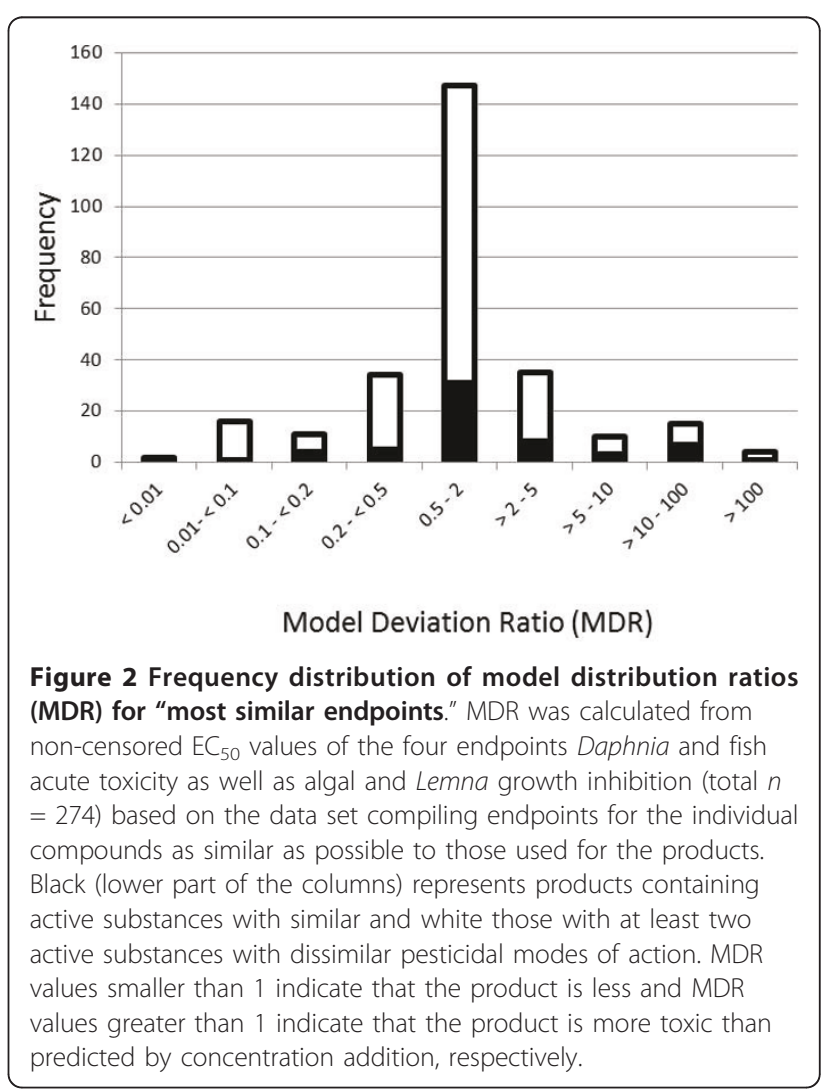




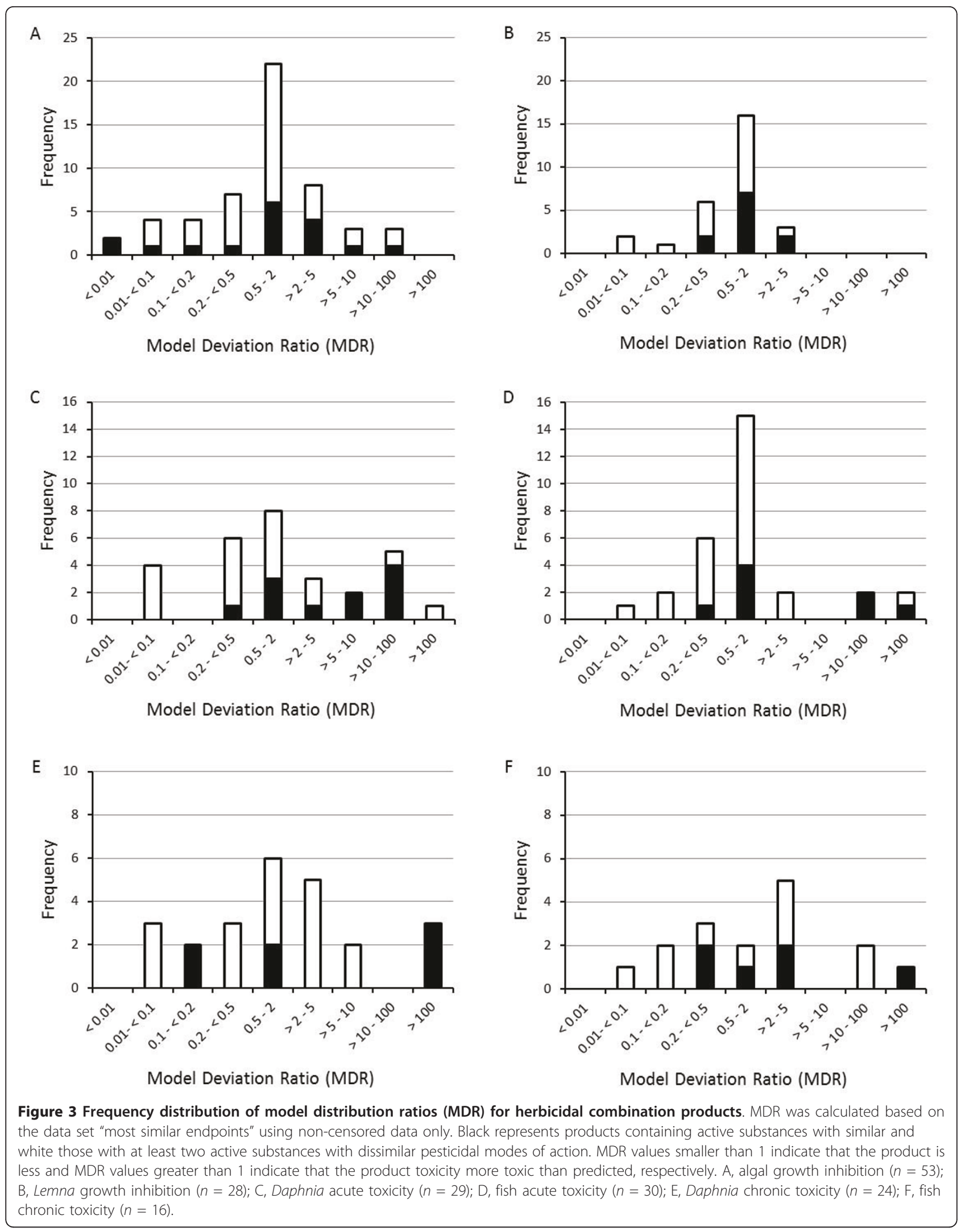


investigated endpoints. Most MDR values fell between 0.5 and 2 for the three endpoints algal growth inhibition (41.5\%), Lemna growth inhibition (57.1\%), and acute toxicity to fish $(50.0 \%)$. The distributions of the relatively few MDR values for chronic Daphnia and fish toxicity were rather flat, but no systematic over-or underestimation of product toxicity was apparent for these predictions based on NOEC values. The median MDR value did not differ significantly among the six endpoints (Mood's median test, $p=0.848$ ). In the case of Lemna, the toxicity of a product was never under-or overestimated by more than factor 100 . Overestimation of product toxicity by more than factor 100 (MDR < 0.01 ) occurred only with regard to algal toxicity. The two products for which this considerable overestimation of algal toxicity was observed contained both the active substance MCPA together with other synthetic auxins. No overestimation of product toxicity by more than factor 100 was found for the endpoints acute and chronic toxicity to both Daphnia and fish, while underestimation of product toxicity by more than factor 100 (MDR $>100$ ) occurred only in these endpoints. There were three products for which more than 100-fold underestimation of acute product toxicity to Daphnia or fish was found. They contained a.s. from four different mode of action groups (synthetic auxins, inhibitors of branched chain amino acid synthesis, inhibitors of photosystems II, and inhibitors of carotenoid synthesis). Yet, there were other products containing the same a.s. or the same mode-of-action combination that had MDR values between 0.5 and 2 for the same endpoints.

\section{Fungicidal combination products}

Figure 4 depicts the frequency distribution of the noncensored MDR values for the fungicidal combination products. The evaluation involved only five endpoints because no data were available for the products regarding Lemna growth inhibition (toxicity to water plants is no standard data requirement for fungicides). The large majority of MDR values fell between 0.5 and 2 for acute toxicity towards Daphnia (79.1\%) and fish $(71.4 \%)$ as well as for algal growth inhibition (44.9\%), while the frequency distribution of MDR values for the (rarely available) endpoints chronic Daphnia and fish toxicity was much flatter. Underestimation of product toxicity occurred most often with regard to algal growth inhibition (median MDR value of 1.34). Yet, no significant difference was detected among the five different endpoints (Mood's median test, $p=0.383$ ).

\section{Explanatory relationships}

Products with two active substances had a median MDR value of $0.990(n=294)$, while the median MDR value of products with three and four active substances (median MDR of 1.5, $n=43$ and median MDR of $0.815, n=$ 4 , respectively) deviated more from 1 , yet in opposite directions. The number of a.s. in a product did not significantly influence the MDR (Mood's median test, $p=$ 0.508). The MDR of the endpoints algal and Lemna growth inhibition was not significantly correlated across herbicidal products. Across fungicidal and herbicidal products, there was also no significant correlation between the MDR of acute and chronic toxicity towards Daphnia, acute and chronic toxicity towards fish, or chronic toxicity towards Daphnia and chronic toxicity towards fish (Spearman rank correlation, all $p>0.05$ ). Daphnia and fish acute toxicity were the only two endpoints that showed a significant correlation between MDR values (Spearman rank correlation, $p<0.05, R=$ $+0.433, n=60$ ). Hence, if the toxicity of a given mixture can be well predicted for one endpoint by CA, this finding cannot be extrapolated to other endpoints, with the exception of acute toxicity in Daphnia and fish.

\section{Frequency of product toxicity underestimation based on "most similar endpoints"}

As mentioned before, in 100 of the 441 comparisons, censored input data did not allow the calculation of a non-censored MDR value. In 42 of these 100 cases, however, it could be deduced that the MDR was greater than a value above 1 (i.e., overestimation of product toxicity can be excluded), and in eight cases, smaller than a value below 1 (i.e., underestimation of product toxicity can be excluded). In the other 58 cases, no meaningful information for the further analysis was obtained (indicated as n.d.).

Table 2 compiles the percentage of cases where the CA prediction underestimates the toxicity of the product by more than a factor of $2,5,10$, or 100 , respectively, based on the data set "most similar endpoints". This compilation includes the $42 \mathrm{MDR}$ values resulting from censored data mentioned above in addition to the MDR values depicted in Figures 3 and 4. Since the majority of censored MDR values occurred for acute toxicity towards Daphnia and fish, particularly for these two endpoints the frequency of product toxicity underestimation increased due to the inclusion of censored data. While the non-censored MDR values indicated that product toxicity underestimation was more frequent for fungicides (see above), the inclusion of censored data indicated that the frequency of underestimation was slightly higher in herbicides than in fungicides. For algal and Lemna growth inhibition, MDR values greater than 100 were not observed at all for herbicidal combination products and the frequency of more than tenfold underestimation was below $10 \%$. For fungicidal combination products, acute toxicity towards Daphnia and fish was rarely underestimated: the MDR was greater than 5 in less than $10 \%$ and greater than 10 in less than $2 \%$ of cases. Hence, the prediction of mixture toxicity by $\mathrm{CA}$ 


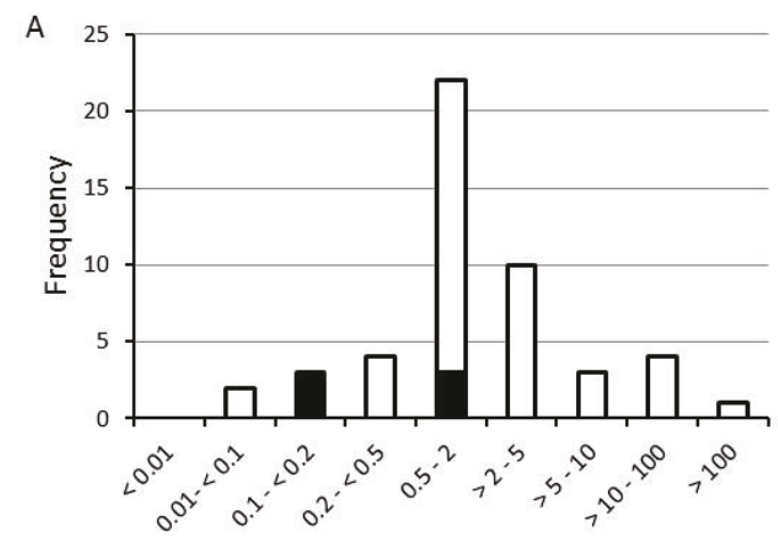

Model Deviation Ratio (MDR)

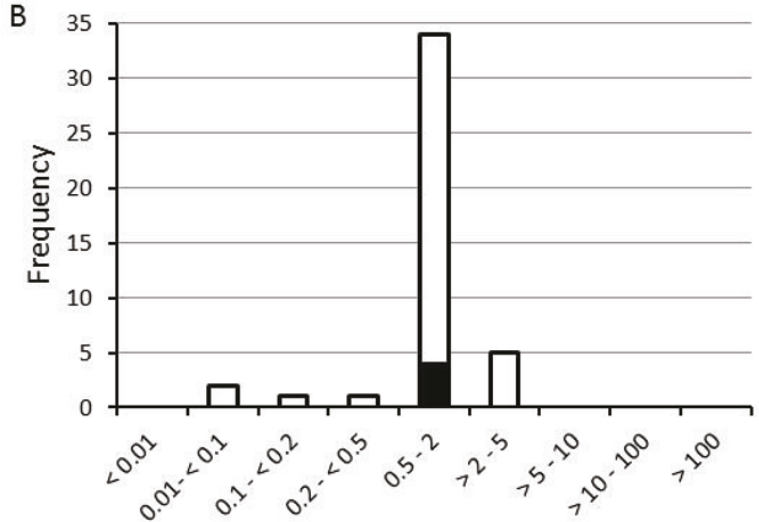

Model Deviation Ratio (MDR)

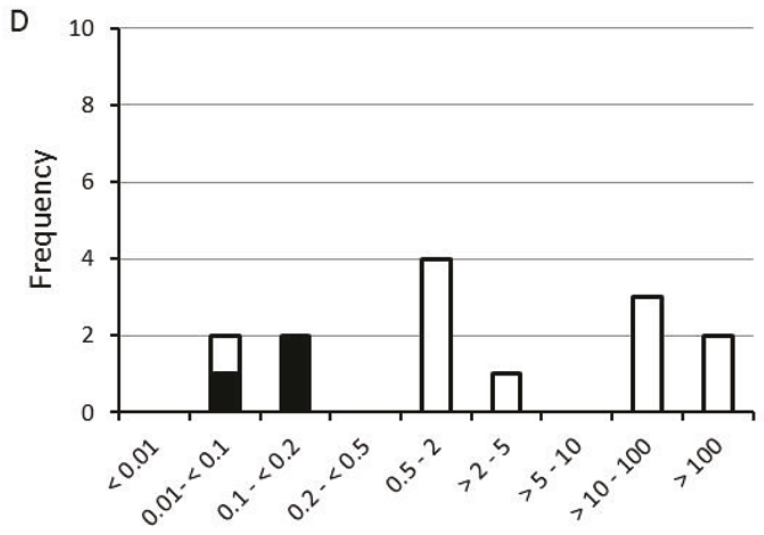

Model Deviation Ratio (MDR)

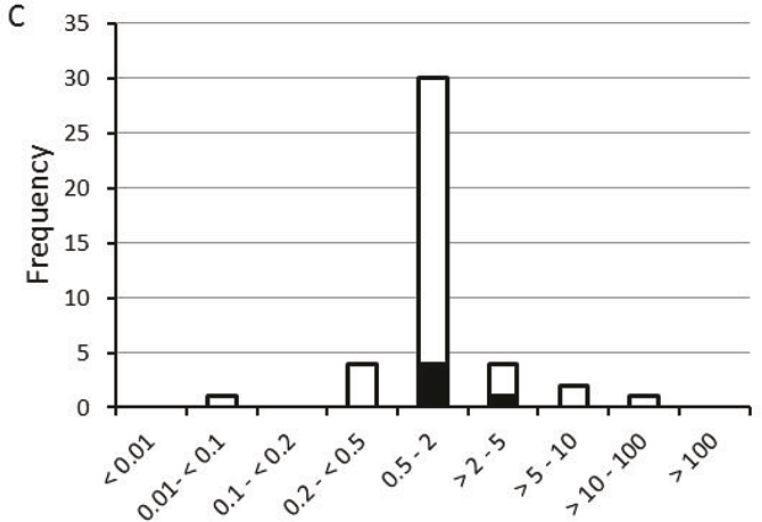

Model Deviation Ratio (MDR)

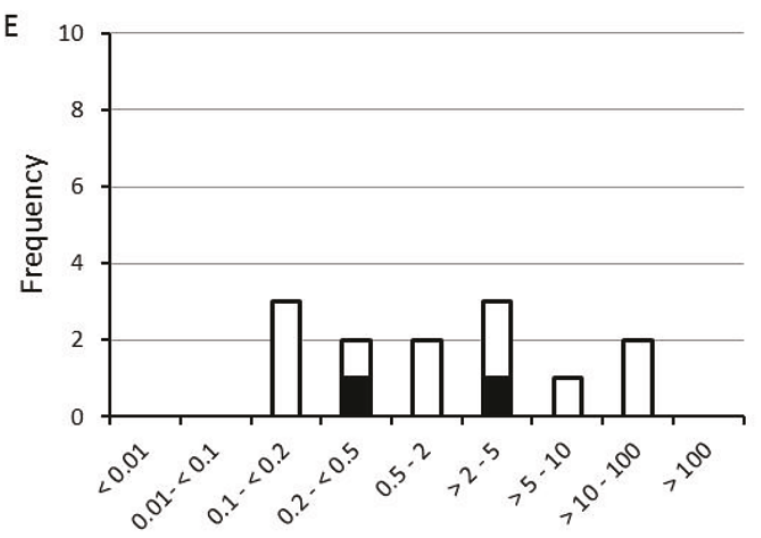

Model Deviation Ratio (MDR)

Figure 4 Frequency distribution of model distribution ratios (MDR) for fungicidal combination products. MDR was calculated based on the data set "most similar endpoints" using non-censored data only. Black represents products containing active substances with similar and white those with at least two active substances with dissimilar pesticidal modes of action. MDR values smaller than 1 indicate that the product is less and MDR values greater than 1 indicate that the product is more toxic than predicted, respectively. A, algal growth inhibition ( $n=49)$; $B$, Daphnia acute toxicity $(n=43)$; C, fish acute toxicity $(n=42)$; D, Daphnia chronic toxicity $(n=14)$; E, fish chronic toxicity $(n=13)$. 
was generally in good agreement with the observed mixture toxicity particularly when the supposedly more sensitive organisms for the respective pesticidal mode of action was assessed, i.e., algae and Lemna as primary producers in the case of herbicides.

In the case of the acute toxicity of herbicidal products towards Daphnia and fish, the product toxicity was underestimated by more than factor 10 in more than $20 \%$ of the analyzed cases (Table 2). The acute toxicity towards either Daphnia or fish was underestimated by more than factor 10 in 17 herbicidal combination products, in 11 of those for both endpoints. Among the 17 products, there were four with more than 100-fold underestimation of Daphnia and fish acute toxicity as well as another five products with more than 100-fold underestimation of either Daphnia or fish acute toxicity. All these nine products had (as far as known) an acute toxicity $\mathrm{EC}_{50}$ of below $1 \mathrm{mg} \sum$ a.s./L for Daphnia and fish, with the exception of one acute fish toxicity $\mathrm{EC}_{50}$ of $1.78 \mathrm{mg} \sum$ a.s./L. The relevance of such a low $\mathrm{EC}_{50}$ value of a product is illustrated by the finding that for three of the above-mentioned nine products the $\mathrm{EC}_{50}$ for both Daphnia and fish was lower than all available $\mathrm{EC}_{50}$ values for aquatic primary producers and would therefore be relevant for the aquatic risk assessment

Table 2 Frequency of underestimating product toxicity based on the data set "most similar endpoints"

\begin{tabular}{|c|c|c|c|c|}
\hline & \multicolumn{4}{|c|}{ Percentage (\%) of MDR } \\
\hline & $>2$ & $>5$ & $>10$ & $>100$ \\
\hline \multicolumn{5}{|l|}{ Algal growth inhibition } \\
\hline Herbicidal products (58) & 25.9 & 12.1 & 6.9 & 0.0 \\
\hline Fungicidal products (51) & 37.2 & 15.7 & 9.8 & 2.0 \\
\hline \multicolumn{5}{|l|}{ Lemna growth inhibition } \\
\hline Herbicidal products (32) & 9.4 & 0.0 & 0.0 & 0.0 \\
\hline \multicolumn{5}{|l|}{ Daphnia acute toxicity } \\
\hline Herbicidal products (59) & 35.6 & 30.5 & 25.4 & 8.5 \\
\hline Fungicidal products (53) & 13.2 & 0.0 & 0.0 & 0.0 \\
\hline \multicolumn{5}{|l|}{ Fish acute toxicity } \\
\hline Herbicidal products (61) & 26.2 & 21.3 & 21.3 & 13.1 \\
\hline Fungicidal products (52) & 19.2 & 9.6 & 1.9 & 0.0 \\
\hline \multicolumn{5}{|l|}{ Daphnia chronic toxicity } \\
\hline Herbicidal products (25) & 44.0 & 24.0 & 16.0 & 12.0 \\
\hline Fungicidal products (16) & 37.5 & 31.3 & 31.3 & 12.5 \\
\hline \multicolumn{5}{|l|}{ Fish chronic toxicity } \\
\hline Herbicidal products (20) & 50.0 & 20.0 & 20.0 & 10.0 \\
\hline Fungicidal products (14) & 50.0 & 28.6 & 21.4 & 0.0 \\
\hline
\end{tabular}

Given is the percentage of model deviation ratios (MDR), including censored and non-censored data, that were determined as being greater than the value of $2,5,10$, or 100 , respectively, for each of the six assessed endpoints using the data set compiling toxicity measures derived in tests with the individual active substances as similar as possible to those conducted with the products. Percentage MDR is related to the number of herbicidal and fungicidal combination products (indicated in parentheses) for which data for the product and all active substances were available for the given endpoint.
(Table 3). The a.s. present in these three products were mostly more toxic to primary producers than to Daphnia or fish (Table 3). Hence, the most sensitive organism group differed between the combination product (most sensitive: heterotrophic organisms) and the a.s. contained therein (most sensitive: primary producers).

\section{Predictions based on "most sensitive endpoints"}

The EU list of endpoints provided for some a.s. no data for some endpoints, e.g., because the endpoint was not triggered during the risk assessment (i.e., chronic invertebrate and fish toxicity) or not relevant in the final risk assessment (i.e., acute toxicity towards invertebrates). The total number of comparisons between predicted and observed product toxicity was therefore lower than when using data from the UBA database. In addition, more cases resulted in a censored or not determinable MDR value. The difference between the MDR values calculated based on the two data sets was significant (Mood's median test, $p=0.0004$ ). Figure 5 illustrates the frequency distribution of the 230 non-censored MDR values obtained from the data set "most sensitive endpoints", excluding NOEC-based calculations. With a median of 0.74 , this MDR frequency distribution is clearly not symmetrical but indicates a systematic overestimation of product toxicity. This confirms that the approach of the "most sensitive endpoints" is the more conservative in comparison to the approach using "most similar endpoints". Yet, there were still a few cases where the product toxicity was underestimated by more than factor 10 .

When taking additionally censored MDR values into account for this data set (Table 4), the frequency of underestimating product toxicity increased. Yet, the frequency of more than tenfold product toxicity underestimation amounted to more than $5 \%$ only when the calculation was based on NOEC values as well as in the case of acute Daphnia and fish toxicity of herbicidal combination products. Among the fungicidal combination products, Daphnia and fish acute toxicity was very rarely underestimated by more than factor 5 and never by more than factor 10 .

\section{Systematic patterns in the deviation between prediction and observation}

An analysis of all MDR values in view of the combined mode-of-action groups revealed only two cases that showed a noticeable pattern in terms of a systematic underestimation of product toxicity. The first case was the combination of iodosulfuron (always present at minor proportion) with other herbicidal a.s. with the same mode of action (inhibitor of branched chain amino acid synthesis). The acute toxicity towards Daphnia or fish of all five assessed products was 
Table 3 Aquatic toxicity of selected herbicidal combination products and the active substances contained therein

\begin{tabular}{|c|c|c|c|c|}
\hline & $\begin{array}{c}\text { Lemna growth } \\
\text { inhibition }\end{array}$ & $\begin{array}{c}\text { Algal growth } \\
\text { inhibition }\end{array}$ & $\begin{array}{l}\text { Acute toxicity to } \\
\text { Daphnia }\end{array}$ & $\begin{array}{l}\text { Acute toxicity to } \\
\text { fish }\end{array}$ \\
\hline 2,4-D (0.473) and Triclopyr (0.527) & $\begin{array}{l}\mathrm{EC}_{50} \text { : n.a. } \\
\text { MDR: n.a. }\end{array}$ & $\begin{array}{l}\mathrm{EC}_{50}: 8.48 \\
\text { MDR: } 3.7\end{array}$ & $\begin{array}{l}E C_{50}: 0.124 \\
\text { MDR: } 66.2\end{array}$ & $\begin{array}{l}L C_{50}: 0.212 \\
\text { MDR: } 314.2\end{array}$ \\
\hline Mecoprop-P (0.617) and loxynil (0.383) & $\begin{array}{l}E C_{50}: 0.685 \\
\text { MDR: } 0.1\end{array}$ & $\begin{array}{l}E C_{50}: 1.72 \\
\text { MDR: } 31.0\end{array}$ & $\begin{array}{c}E C_{50}: 0.057 \\
M D R:>150.6\end{array}$ & $\begin{array}{l}\text { LC } 50: 0.180 \\
\text { MDR: } 115.1\end{array}$ \\
\hline $\begin{array}{l}\text { Fluroxypyr (0.333) and loxynil (0.333) and Bromoxynil } \\
(0.333)\end{array}$ & $\begin{array}{l}E C_{50}: 0.147 \\
\text { MDR: n.a. }\end{array}$ & $\begin{array}{l}E C_{50}: 2.02 \\
\text { MDR: } 0.9\end{array}$ & $\begin{array}{l}\mathrm{EC}_{50}: 0.020 \\
\mathrm{MDR}:>394.5\end{array}$ & $\begin{array}{l}L C_{50}: 0.072 \\
M D R:>243.2\end{array}$ \\
\hline $2,4-D(O)$ & n.a. & 24.2 & 4.0 & 45.0 \\
\hline Triclopyr (O) & n.a. & 42.0 & 132.9 & 117.0 \\
\hline Mecoprop-P (O) & 1.57 & 223.1 & $>100$ & 198.3 \\
\hline Fluroxypyr (O) & n.a. & 49.8 & $>100$ & $>100$ \\
\hline loxynil (C) & 0.027 & 24.0 & 3.5 & 8.5 \\
\hline Bromoxynil (C) & 0.033 & 0.65 & 12.5 & 23.0 \\
\hline
\end{tabular}

Given are $\mathrm{E}(\mathrm{L}) \mathrm{C}_{50}$ values in relation to the summed concentration of the a.s. in the product (milligrams of the sum of a.s. per liter, mg $\Sigma$ a.s./L) of the three herbicidal combination products with high toxicity and a model deviation ratio (MDR) greater than 100 for acute toxicity to Daphnia or fish (based on the data set "most similar endpoints"). In addition, the toxicity of the active substances ( $E C_{50}$ in milligrams of a.s. per liter) contained in these products is provided as well as their herbicidal mode of action (in parentheses) according to HRAC [16]: O, synthetic auxin; C, inhibitor of photosystem II. n.a. not available.

considerably underestimated by the CA prediction (Table 5).

The second case was products that combined fungicides from the Fungicide Resistance Action Committee (FRAC) groups G2 and G1, i.e., fungicides that inhibit different enzymes in the ergosterol biosynthesis, and sometimes additionally fungicides from another group. The MDR values calculated for these 11 combination

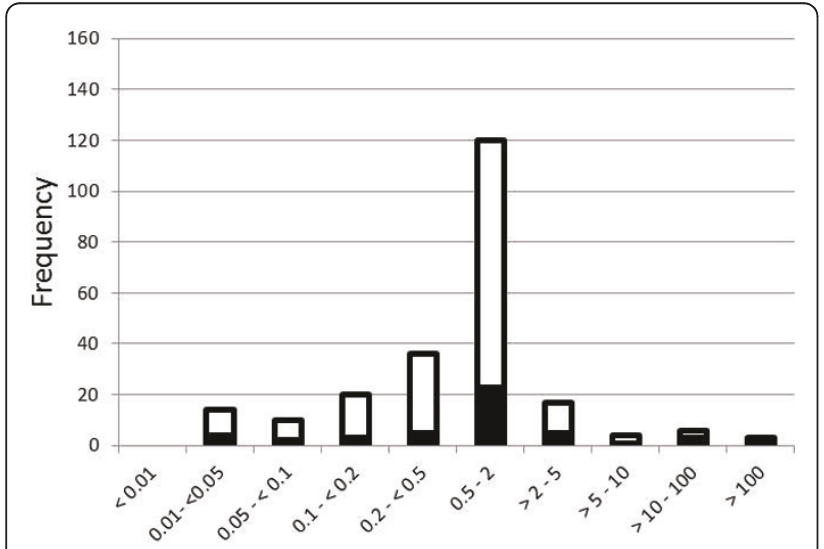

Model Deviation Ratio (MDR)

Figure 5 Frequency distribution of model distribution ratios (MDR) for "most sensitive endpoints". MDR was calculated from non-censored $\mathrm{EC}_{50}$ values of the four endpoints Daphnia and fish acute toxicity as well as algal and Lemna growth inhibition (total $n$ $=230$ ) based on the data set compiling the lowest toxicity measures within each endpoint. Black represents products containing active substances with similar and white those with at least two active substances with dissimilar pesticidal modes of action. MDR values smaller than 1 indicate that the product is less and MDR values greater than 1 indicate that the product is more toxic than predicted by concentration addition, respectively. products are shown in Table 6 (only for the data set "most similar endpoints") together with the $\mathrm{EC}_{50}$ values of the products and the individual a.s. (input data for CA prediction). The toxicity of the products towards algae was underestimated by more than factor 2 and up to more than factor 100 in all products that contained fenpropidin or fenpropimorph, but was not underestimated in the two products that contained spiroxamine. No systematic deviation of the prediction was observed for these 11 products with regard to acute fish or Daphnia toxicity. Algae were more sensitive than Daphnia or fish for each of the three G2 a.s. This holds also for most of the other fungicidal a.s. contained in these products. Hence, in contrast to the case of products that contained iodosulfuron described above, the most sensitive organism group (heterotrophic organisms) for these fungicidal products did not differ between the combination product and the individual a.s. contained therein. Azoles were present in $54.5 \%$ of all assessed fungicidal combination products, but with the exception of the G1/G2 combination mentioned above, there was no apparent systematic pattern of product toxicity underestimation to any of the assessed endpoints with regard to the presence of azoles.

\section{Discussion}

Compliance between the predicted mixture toxicity and the observed toxicity of the combination product was overall very good in the present study, with the majority of cases (53.6\% in the first data set) showing a less than twofold deviation as long as the predictions were based on non-censored $\mathrm{EC}_{50}$ values. Yet, two previous studies $[14,15]$ that reviewed available literature on mixture toxicity experiments reported a much higher percentage of cases with such a degree of compliance. Deneer [14] 
Table 4 Frequency of underestimating product toxicity based on the data set "most sensitive endpoints"

\begin{tabular}{|c|c|c|c|c|}
\hline & \multicolumn{4}{|c|}{ Percentage (\%) of MDR } \\
\hline & $>2$ & $>5$ & $>10$ & $>100$ \\
\hline \multicolumn{5}{|l|}{ Algal growth inhibition } \\
\hline Herbicidal products (58) & 12.1 & 6.9 & 3.4 & 1.7 \\
\hline Fungicidal products (45) & 20.0 & 11.1 & 4.4 & 0.0 \\
\hline \multicolumn{5}{|c|}{ Water plant growth inhibition } \\
\hline Herbicidal products (35) & 8.6 & 5.7 & 2.9 & 0.0 \\
\hline \multicolumn{5}{|c|}{ Aquatic invertebrate acute toxicity } \\
\hline Herbicidal products (44) & 27.3 & 22.7 & 18.2 & 9.1 \\
\hline Fungicidal products (52) & 9.6 & 0.0 & 0.0 & 0.0 \\
\hline \multicolumn{5}{|l|}{ Fish acute toxicity } \\
\hline Herbicidal products (61) & 23.0 & 19.7 & 18.0 & 6.6 \\
\hline Fungicidal products (51) & 11.8 & 2.0 & 0.0 & 0.0 \\
\hline \multicolumn{5}{|c|}{ Aquatic invertebrate chronic toxicity } \\
\hline Herbicidal products (26) & 42.3 & 23.1 & 19.2 & 3.8 \\
\hline Fungicidal products (12) & 33.3 & 33.3 & 33.3 & 16.7 \\
\hline \multicolumn{5}{|l|}{ Fish chronic toxicity } \\
\hline Herbicidal products (20) & 20.0 & 10.0 & 10.0 & 5.0 \\
\hline Fungicidal products (9) & 11.1 & 0.0 & 0.0 & 0.0 \\
\hline
\end{tabular}

Given is the percentage of model deviation ratios (MDR), including censored and non-censored data, that were determined as being greater than the value of $2,5,10$, or 100 , respectively, for each of the six different endpoints using the data set compiling the lowest toxicity measures for the individual a.s. within each endpoint. Percentage MDR is related to the number of herbicidal and fungicidal combination products (indicated in parentheses) for which data for the product and all active substances were available for the given endpoint.

assessed 26 studies with aquatic organisms, involving 202 mixtures of pesticides, and found that in more than $90 \%$ of cases the CA prediction deviated by less than factor 2 from the measured toxicity of the mixture, with a maximum deviation of factor 20 . Insecticides tested in fish and crustaceans as well as herbicides tested in algae represented the by far largest part of the studies assessed by [14], while fungicides were hardly covered at all (about 3\%). Belden et al. [15] summarized 207 experiments with mixtures of pesticides explicitly testing CA in aquatic organisms. In $88 \%$ of these experiments, CA prediction and measured mixture toxicity differed by less than factor 2 , with maximum deviations being less than factor 10. Herbicides and insecticides were also heavily dominating the database analyzed by [15]: there was only one experiment with fungicides among the 122 of the $207 \mathrm{CA}$ experiments that involved combinations of pesticides from the same usage class (e.g., herbicides). Furthermore, the diversity among the 92 insecticidal or herbicidal combinations with similar modes of actions was relatively low in the study of Belden et al. [15] with two different pesticidal modes of actions for the insecticidal and six for the herbicidal combinations.

There are several factors that will contribute to the more frequent occurrence and larger degree of deviation in the present study compared to these previous studies $[14,15]$ : the types of pesticide mixtures considered, the heterogeneity of the input data used for the CA prediction, the bias potentially introduced by using the CA concept also for (supposedly) dissimilarly acting substances, synergistic interactions between active substances, and influence of formulation additives. These factors and their potential impact on the predictability of the mixture toxicity will be discussed in the following.

\section{Represented mixtures of pesticides}

In the present study, the MDR values for fungicidal combination products were significantly larger than

Table 5 Aquatic toxicity of combination products with iodosulfuron and of the active substances contained therein

\begin{tabular}{|c|c|c|c|c|}
\hline & $\begin{array}{l}\text { Lemna growth } \\
\text { inhibition }\end{array}$ & $\begin{array}{l}\text { Algal growth } \\
\text { inhibition }\end{array}$ & $\begin{array}{l}\text { Acute toxicity to } \\
\text { Daphnia }\end{array}$ & $\begin{array}{l}\text { Acute toxicity } \\
\text { to fish }\end{array}$ \\
\hline Iodosulfuron (0.030) and Foramsulfuron (0.970) & $\begin{array}{l}\mathrm{EC}_{50}: 0.001 \\
\mathrm{MDR}: 0.57\end{array}$ & $\begin{array}{l}\mathrm{EC}_{50}: \text { n.a. } \\
\text { MDR: n.a. }\end{array}$ & $\begin{array}{l}\mathrm{EC}_{50}: 0.102 \\
\mathrm{MDR}:>982\end{array}$ & $\begin{array}{l}\mathrm{LC}_{50}: 0.243 \\
\mathrm{MDR}:>412\end{array}$ \\
\hline lodosulfuron (0.031) and Foramsulfuron (0.969) & $\begin{array}{l}\mathrm{EC}_{50}: 0.0008 \\
\text { MDR: } 0.85\end{array}$ & $\begin{array}{l}\mathrm{EC}_{50}: 0.229 \\
\mathrm{MDR}: 7.77\end{array}$ & $\begin{array}{l}\mathrm{EC}_{50}: \text { n.a. } \\
\text { MDR: n.a. }\end{array}$ & $\begin{array}{l}\mathrm{LC}_{50}: 0.805 \\
\mathrm{MDR}:>124\end{array}$ \\
\hline Iodosulfuron (0.085) and Amidosulfuron (0.915) & $\begin{array}{l}\mathrm{EC}_{50}: 0.0015 \\
\text { MDR: } 4.43\end{array}$ & $\begin{array}{l}\mathrm{EC}_{50}: 0.359 \\
\mathrm{MDR}: 2.07\end{array}$ & $\begin{array}{l}\mathrm{EC}_{50}: 0.437 \\
\mathrm{MDR}:>130\end{array}$ & $\begin{array}{l}\mathrm{LC}_{50}: 1.78 \\
\mathrm{MDR}:>151\end{array}$ \\
\hline $\begin{array}{l}\text { lodosulfuron (0.039) and Amidosulfuron (0.299) and } \\
\text { Propoxycarbazone (0.662) }\end{array}$ & $\begin{array}{c}\mathrm{EC}_{50}: 0.0032 \\
\text { MDR: } 1.87\end{array}$ & $\begin{array}{l}\mathrm{EC}_{50}: \text { n.a. } \\
\text { MDR: n.a. }\end{array}$ & $\begin{array}{c}\mathrm{EC}_{50}: 7.94 \\
\mathrm{MDR}:>10.4\end{array}$ & $\begin{array}{c}\mathrm{LC}_{50}: 5.67 \\
\text { MDR: > } 18.1\end{array}$ \\
\hline Iodosulfuron (0.055) and Propoxycarbazone (0.945) & $\begin{array}{c}\mathrm{EC}_{50}: 0.006 \\
\text { MDR: } 0.84\end{array}$ & $\begin{array}{l}\mathrm{EC}_{50}: 1.63 \\
\text { MDR: } 0.44\end{array}$ & $\begin{array}{c}E_{50}: 5.29 \\
M D R:>20.5\end{array}$ & $\begin{array}{c}\mathrm{LC}_{50}: 2.21 \\
\text { MDR: > } 36.2\end{array}$ \\
\hline lodosulfuron & 0.00083 & $0.064(0.07)^{a}$ & $>100$ & $>100$ \\
\hline Foramsulfuron & 0.00065 & 12.5 & $>100$ & $>100$ \\
\hline Amidosulfuron & 0.0176 & 47.0 & 55.0 & $>320$ \\
\hline Propoxycarbazone & 0.0064 & 1.57 & $>107$ & $>77.2$ \\
\hline
\end{tabular}

Given are $\mathrm{E}(\mathrm{L}) \mathrm{C}_{50}$ values (milligrams of the sum of a.s. per liter, $\mathrm{mg}$ La.s./L) of herbicidal combination products that contain iodosulfuron and their model deviation ratio (MDR), based on the data set "most similar endpoints". In addition, the toxicity of all active substances (EC ${ }_{50}$ in milligrams of a.s. per liter) contained in these products is provided. All a.s. in these products have the same herbicidal mode of action (inhibitor of branched chain amino acid synthesis). n.a., no data available for the product. ${ }^{a}$ Value from another test used for the last product 
Table 6 Aquatic toxicity of combination products with fungicides that inhibit different enzymes in the ergosterol biosynthesis

\begin{tabular}{|c|c|c|c|}
\hline & $\begin{array}{c}\text { Algal growth } \\
\text { inhibition }\end{array}$ & $\begin{array}{c}\text { Acute toxicity to } \\
\text { Daphnia }\end{array}$ & $\begin{array}{l}\text { Acute toxicity to } \\
\text { fish }\end{array}$ \\
\hline Fenpropidin (0.600) and Tebuconazole (0.200) and Propiconazole (0.200) & $\begin{array}{l}\mathrm{EC}_{50}: 0.000088 \\
\text { MDR: } 107.8\end{array}$ & $\begin{array}{l}\mathrm{EC}_{50}: 4.39 \\
\text { MDR: } 1.70\end{array}$ & $\begin{array}{l}\text { LC }_{50}: 1.82 \\
\text { MDR: } 1.80\end{array}$ \\
\hline Fenpropidin (0.783) and Propiconazole (0.217) & $\begin{array}{c}\mathrm{EC}_{50}: 0.00019 \\
\text { MDR: } 37.9\end{array}$ & $\begin{array}{l}\mathrm{EC}_{50}: 4.46 \\
\text { MDR: } 1.51\end{array}$ & $\begin{array}{l}\text { LC } 50: 1.92 \\
\text { MDR: } 1.83\end{array}$ \\
\hline Fenpropidin (0.789) and Difenoconazole (0.211) & $\begin{array}{c}\mathrm{EC}_{50}: 0.00016 \\
\text { MDR: } 45.6\end{array}$ & $\begin{array}{l}\mathrm{EC}_{50}: 2.97 \\
\text { MDR: } 0.84\end{array}$ & $\begin{array}{l}\text { LC }_{50}: 1.24 \\
\text { MDR: } 1.60\end{array}$ \\
\hline Fenpropimorph (0.749) and Epoxiconazole (0.251) & $\begin{array}{l}E C_{50}: 1.68 \\
M D R:>2.0\end{array}$ & $\begin{array}{l}\mathrm{EC}_{50}: 1.44 \\
\text { MDR: } 1.91\end{array}$ & $\begin{array}{l}L^{L} C_{50}: 0.720 \\
\text { MDR: } 6.04\end{array}$ \\
\hline Fenpropimorph (0.577) and Epoxiconazole (0.116) and Pyraclostrobin (0.307) & $\begin{array}{l}\mathrm{EC}_{50}: 0.107 \\
\text { MDR: } 2.41\end{array}$ & $\begin{array}{l}E_{50}: 0.048 \\
\text { MDR: } 1.05\end{array}$ & $\begin{array}{l}\text { LC } 50: 0.028 \\
\text { MDR: } 0.75\end{array}$ \\
\hline Fenpropimorph (0.375) and Epoxiconazole (0.313) and Kresoxim-methyl (0.313) & $\begin{array}{l}\mathrm{EC}_{50}: 0.024 \\
\text { MDR: } 6.48\end{array}$ & $\begin{array}{l}\mathrm{EC}_{50}: 0.168 \\
\text { MDR: } 3.16\end{array}$ & $\begin{array}{l}L^{L} C_{50}: 0.665 \\
\text { MDR: } 0.74\end{array}$ \\
\hline Fenpropimorph (0.656) and Epoxiconazole (0.172) and Kresoxim-methyl (0.172) & $\begin{array}{l}\mathrm{EC}_{50}: 0.050 \\
\text { MDR: } 4.08\end{array}$ & $\begin{array}{l}\mathrm{EC}_{50}: 0.385 \\
\text { MDR: } 2.10\end{array}$ & $\begin{array}{l}\text { LC }_{50}: 1.55 \\
\text { MDR: } 0.53\end{array}$ \\
\hline $\begin{array}{l}\text { Fenpropimorph (0.555), Epoxiconazole (0.148), Kresoxim-methyl (0.148), } \\
\text { Quinoxyfen (0.148) }\end{array}$ & $\begin{array}{l}\mathrm{EC}_{50}: 0.005 \\
\text { MDR: } 22.3\end{array}$ & $\begin{array}{l}\mathrm{EC}_{50}: 0.260 \\
\text { MDR: } 1.32\end{array}$ & $\begin{array}{l}\text { LC } 50: 2.01 \\
\text { MDR: } 0.31\end{array}$ \\
\hline Fenpropimorph (0.593) and Epoxiconazole (0.185) and Metrafenone (0.222) & $\begin{array}{l}\mathrm{EC}_{50}: 0.161 \\
\text { MDR: } 2.73\end{array}$ & $\begin{array}{l}\mathrm{EC}_{50}: 1.93 \\
\mathrm{MDR}:>0.98\end{array}$ & $\begin{array}{l}\mathrm{LC}_{50}: 2.33 \\
\text { MDR: }>0.96\end{array}$ \\
\hline Spiroxamine (0.652) and Prothioconazole (0.348) & $\begin{array}{l}\mathrm{EC}_{50}: 0.007 \\
\text { MDR: } 1.18\end{array}$ & $\begin{array}{l}\mathrm{EC}_{50}: 2.96 \\
\text { MDR: } 0.90\end{array}$ & $\begin{array}{l}\text { LC }_{50}: 3.08 \\
\text { MDR: } 1.44\end{array}$ \\
\hline Spiroxamine (0.653) and Tebuconazole (0.347) & $\begin{array}{l}\mathrm{EC}_{50}: 0.009 \\
\text { MDR: } 0.53\end{array}$ & $\begin{array}{l}\mathrm{EC}_{50}: 3.67 \\
\text { MDR: } 2.00\end{array}$ & $\begin{array}{l}\text { LC }_{50}: 3.81 \\
\text { MDR: } 2.93\end{array}$ \\
\hline Fenpropidin (G2) & 0.0057 & 6.15 & $2.57(3.25)^{\mathrm{a}}$ \\
\hline Fenpropimorph (G2) & $16.8(0.327)^{\mathrm{a}}$ & 2.24 & 5.0 \\
\hline Spiroxamine (G2) & $0.005(0.003)^{\mathrm{a}}$ & 6.1 & 18.5 \\
\hline Tebuconazole (G1) & 1.96 & 11.8 & 6.4 \\
\hline Propiconazole (G1) & 0.6 & 10.2 & 4.9 \\
\hline Difenoconazole (G1) & 1.2 & 0.77 & 0.81 \\
\hline Epoxiconazole (G1) & $>1(1.19)^{a}$ & 8.69 & 3.14 \\
\hline Pyraclostrobin (C3) & 0.152 & 0.016 & 0.065 \\
\hline Kresoxim-methyl (C) & 0.063 & 0.186 & 168.0 \\
\hline Quinoxyfen (E1) & 0.0031 & 0.08 & 0.27 \\
\hline Metrafenone $(U)$ & 0.711 & $>0.92$ & $>0.82$ \\
\hline Prothioconazole (G1) & 1.1 & 1.3 & 1.83 \\
\hline
\end{tabular}

Given are $\mathrm{E}(\mathrm{L}) \mathrm{C}_{50}$ values in relation to the summed concentration of the a.s. in the product (milligrams of the sum of a.s. per liter) of 11 fungicidal combination products (based on the data set "most similar endpoints"). In addition, the toxicity of the active substances (EC 50 in milligrams of a.s. per liter) contained in these products is provided as well as their fungicidal mode of action (in parentheses) according to FRAC [17]: G2, inhibitor of $\Delta 14-$-reductase and $\Delta 8$ to $\Delta 7$-isomerase in the ergosterol biosynthesis; G1, inhibitor of C14-demethylase in the ergosterol biosynthesis; C3, inhibitor of mitochondrial respiration; E1, growth signal disruptor; $\mathrm{U}$, inhibitor of sporulation or mycelium development. n.a. no data available for product. ${ }^{a}$ Values from different tests (e.g., $\mathrm{EC}_{50}$ based on growth rate or biomass) selected for different products.

those of herbicidal products (as long as censored data were excluded) and almost half of the database consisted of fungicidal combinations. The strong underrepresentation of fungicides in previous meta-studies [14,15] may therefore at least partly explain their finding of a higher compliance between CA prediction and mixture toxicity observation. Furthermore, the highest frequency of MDR values above 1 were found for fungicidal combinations tested with algae and herbicidal combinations tested with fish or Daphnia. These are exactly the kind of mixtures hardly represented in the literature $[14,15]$.

On the other hand, no combination products with insecticides were assessed in the present study (as very few are authorized in Germany), whereas mixtures with insecticides were found to deviate most frequently from CA [14]. The databases of both previous studies were presumably biased towards a higher likelihood of finding large MDR values, because many of the reviewed experiments aimed at detecting interactions, particularly synergistic interactions, and the tested pesticide combinations were accordingly selected. Hence, the representation of certain pesticide usage classes, modes of action and test species combinations in the reviewed studies $[14,15]$ may have considerably influenced the degree and frequency of detected deviations from CA prediction in both directions. 


\section{Heterogeneity of the data used for mixture toxicity predictions}

A key pre-condition for reliable CA predictions is that similar endpoints are assessed for the mixture and all individual components, i.e., the identity of test species, exposure design and measured toxic effects, with tests being ideally conducted in parallel in the same experimental setting. These assumptions were presumably fulfilled by all experiments reviewed by [14] and [15]. A statistical simulation study showed that the likelihood of detecting a deviation from $\mathrm{CA}$ although the mixture components act truly concentration-additive (i.e., the false positive rate) increases with increasing variability in the test population sensitivity among the tests performed with the mixture and its individual components [18]. With regard to the calculated MDR values, this means that the more the considered toxicity tests vary in factors such as usage of different test species, test protocols, exposure conditions, differences between nominal and measured test substance concentrations and measured toxic effects, the greater will be the deviations (i.e., the larger the absolute MDR values). As long as the heterogeneity is randomly distributed, which can be assumed for the here conducted calculations, it will not lead to a bias, i.e., the occurrence of systematic under-or overestimation of product toxicity. Hence, the large heterogeneity in the input data used for the mixture toxicity predictions in the present study explains a great deal of the observed deviations from CA prediction, and the clear finding of a symmetric MDR frequency distribution in the first data set agrees with the expectation of a non-biased increase only in the range of the MDR values.

NOEC values as toxicity measures derived by hypothesis testing depend strongly on the statistical design of a study (e.g., the number of selected test concentrations and replicates), but they do not relate to an identical fixed-effect level across studies with identical designs [19]. Yet, the CA concept assumes that the effect concentrations used for the individual components in the mixture relate to a defined fixed-effect level, e.g., 50\% inhibition. The large and frequent deviations between predicted and measured chronic Daphnia and fish toxicity of the combination products in the present study demonstrate that the use of NOEC values results in a much higher unreliability of mixture toxicity predictions in comparison to using $\mathrm{EC}_{\mathrm{x}}$ values as input data. The use of $\mathrm{EC}_{\mathrm{x}}$ values for the growth inhibition of Lemna and algae (a chronic endpoint) resulted in an overall good compliance between mixture toxicity prediction and observation. This indicates that the reason of unreliable predictions for chronic Daphnia and fish toxicity is not a per se non-predictability of chronic effects by mixture toxicity concepts but rather due to the violation of the fixed-effect level assumption of the CA concept by using NOEC values. As heterogeneity in the input data decreases the reliability of the mixture toxicity prediction, it could be argued that the large variability particularly in the chronic fish studies caused the poor compliance. However, using NOEC values from highly standardized $D$. magna reproduction tests (relatively low data heterogeneity) achieved a similar frequency distribution of MDR values as for chronic fish toxicity. Overall, the use of NOEC data for CA-based mixture toxicity predictions is not advocated.

\section{Similarity in the mode of action}

IA is the appropriate conceptual assumption for predicting the joint toxicity of mixtures composed of substances with dissimilar modes of toxic action $[8,20]$, while the CA prediction will often overestimate the toxicity for such mixtures $[4,9,21,22]$. As deduced theoretically, the mixture toxicity prediction of the IA and the CA concept can deviate at most by factor $n$, with $n$ being the number of mixture components [4]. Hence, the difference between the prediction concepts cannot explain much of the deviation between predicted and measured mixture toxicity observed here for the investigated combination products, which contained mostly two and at maximum four components (i.e., active substances).

Because extensive information regarding both pharmacodynamics and pharmacokinetics is needed to actually judge the similarity of the mode of action of two or more chemicals [23], the preference of IA above CA based on the known or assumed mode of action has been questioned [23-26]. The classification of similar and dissimilar modes of action as applied in the present study relates to the pesticidal mode of action of the a.s., hence to the specific toxicity towards the target organisms based on the known molecular target and the development of cross-resistances in target organisms. No difference in the frequency distribution of MDR values was found between products with similarly and dissimilarly acting a.s. based on this pesticidal mode-ofaction classification. Although the low percentage of products that contained a.s. with similar pesticidal modes of action among the investigated products may have limited the ability to detect systematic differences, the absence of differences indicates that the here assumed dissimilarity of the mode of action did overall not interfere with the applicability of CA. The available information about the mode of action of pesticidal a.s. in non-target organisms is generally limited and thereby hardly supporting a sound decision about the choice between IA and CA. Toxicity may be imposed by the same mode of action particularly when the targeted molecules mediate identical functions in the target organisms and the species used in the ecotoxicological 
testing, e.g., photosynthesis in weeds as target organisms of herbicides and algae or higher aquatic plants as nontarget organisms. For other pesticidal a.s., the mode of action may differ between target and non-target organisms. Baseline toxicity (narcosis), i.e., a similar mode of action, is often assumed in the absence of documented specific toxicity, as for example regarding the toxicity of photosynthesis inhibitors towards heterotrophic organisms $[8,14]$. Another example are de-methylation inhibitors (DMI fungicides, FRAC group G1) that are designed to interfere with cytochrome P450 monooxygenases in target fungi but can affect diverse and different biochemical pathways in various species and thereby display different modes of actions in non-target organisms [27]. These authors reported that in Daphnia magna, DMI fungicides from four different chemical classes were found to exhibit baseline toxicity as well as specific toxicity. The latter differed among substances, i. e., fenarimol and triadimefon affected embryonic eye development while pyrifenox affected molting [27]. Likewise, Ankley et al. [28] reported multiple modes of actions of the DMI fungicides prochloraz and fenarimol in fish. A classification of the similarity of the mode of action strictly with regard to the non-target test species in question would therefore likely result in a different classification of similarity and dissimilarity for these a.s. than that solely based on the pesticidal mode of action. In theory, this could allow detecting a bias related to the default application of the CA concept. However, the necessary detailed information about the modes of action of pesticides in various non-target test species will hardly ever be available, which precludes a verification of this hypothesis.

Overall, the results of the present study further support the use of CA as a pragmatic and reasonably protective approach for predicting the joint aquatic toxicity of pesticide mixtures irrespectively of the known or assumed mode of action of the mixture components [13] for different reasons: (a) from a pragmatic point of view, the available data from the environmental risk assessment only enable the use of the CA but not, at a reasonable effort, the IA concept; (2) the available information on the a.s. does not support a decision about similarity or dissimilarity of their mode of action in non-target organisms and thereby an informed choice between the IA and CA concept; (3) no bias in terms of a systematic overestimation of the joint toxicity of combination products with (supposedly) dissimilar acting a.s was apparent in the investigated data base of 119 combination products.

\section{Underestimation of product toxicity by CA: indication of synergistic interactions?}

Some combination products were identified here as showing deviations from the prediction that may indicate synergistic interactions in specific endpoints. In the following, possible explanations will be discussed, taking also into account the potential influence of formulation additives.

\section{Herbicidal combination products with systematic or high underestimation of product toxicity}

All a.s. in the five products containing iodosulfuron have the same pesticidal mode of action, i.e., they are inhibitors of branched chain amino acid synthesis (ALS herbicides, Herbicide Resistance Action Committee (HRAC) group B). This mode of action is very specific and only of relevance in primary producers, because the targeted enzyme acetolactate is not present in insects, mammals, or other animals [29]. For algae and Lemna, the mixture toxicity predictions met the reported product toxicity with less than tenfold deviation. This finding is in accordance with toxicity experiments conducted in algae with mixtures of herbicides from one homogenous mode-ofaction group such as chloroacetanilide [30] or phenylurea [31] and from diverse mode-of-action groups [32]. In contrast, the acute joint toxicity towards Daphnia and fish of all (assessable) products with iodosulfuron was at least tenfold underestimated. Despite the identical, highly specific herbicidal mode of action of all a.s., a synergistic interaction towards heterotrophic organisms cannot completely be ruled out as a reason for the underestimation of product toxicity. Assuming that the $\mathrm{EC}_{50}$ values for all five combination products are robust, the systematic underestimation may alternatively be explained by inappropriate or qualitatively poor $\mathrm{EC}_{50}$ values of the a.s. for Daphnia and fish (reported for iodosulfuron in both used databases as $>100 \mathrm{mg} / \mathrm{L}$ ). Yet, there are other possible explanations.

Formulation additives can be genuinely toxic [3] but they were not considered here in the mixture toxicity predictions due to a lack of information on their identity and aquatic toxicity. A PPP that contained only iodosulfuron and served as representative formulation for the EU risk assessment procedure was considerably (more than 100-fold) more acutely toxic to Daphnia and fish than the technical iodosulfuron [33]. This may indicate that iodosulfuron is generally formulated with additives that are either toxic or that enhance the toxicity of the a.s. by other mechanisms. Such mechanisms may consist in an influence on the pharmacokinetic of the a.s., for example by promoting uptake into organisms. Examples have been reported for different chemical groups [34] and particularly for certain formulation additives (penetration agents) of herbicidal products [35].

The mixture components with the highest toxicity contribute most or even dominate the toxicity of mixtures in terms of toxic units [4]. The presence of toxic additives in a PPP that are not considered in the mixture toxicity prediction may therefore particularly 
explain underestimation of product toxicity when the test organisms are rather insensitive to the a.s. contained in the formulated product. For organisms that are very sensitive to the a.s., the toxicity of the a.s. will largely dominate the product toxicity, while the in comparison likely rather low toxicity of formulation additives will not contribute much to the mixture toxicity. This explanation fits not only with the observation for the iodosulfuron-containing products but also with the observation for the other herbicidal combination products that showed a considerable underestimation of acute Daphnia and fish toxicity of the product (some are listed in Table 3). While the a.s. contained in these products were almost always more toxic to primary producers than to Daphnia and fish, the latter organisms were more sensitive for the formulated products than primary producers. This supports the general conclusion, that not synergistic interactions between a.s. on a molecularmechanistic basis were the underlying reason, but that it were rather formulation additives that caused the morethan-additive acute toxicity of these herbicidal combination products towards Daphnia and fish.

\section{Fungicidal combination products containing G2 and G1 fungicides}

For combination products containing fenpropidin or fenpropimorph (FRAC group G2) together with DMI fungicides (FRAC group G1) all determined MDR values fell clearly above 1 with regard to algal growth inhibition, but not with regard to acute toxicity towards Daphnia or fish. Fungicides from both groups (G1 and G2) belong to the larger group of sterol biosynthesis inhibitors (SBI fungicides) but they inhibit different key enzymes in the ergosterol biosynthesis of fungi. G2 fungicides inhibit to various degrees $\Delta^{14}$-reductase and $\Delta^{8}$ $\Delta^{7}$-isomerase, while G1 fungicides inhibit C14-demethylase. The exact molecular site of action of SBI fungicides, particularly fenpropidin and fenpropimorph, in algae is not known currently.

The algal toxicity of the fungicidal combination products in question was rather high $\left(\mathrm{EC}_{50}\right.$ below $1 \mathrm{mg} \Sigma$ a.s./L in many cases), which underlines the potential environmental relevance of this finding. It is interesting to note that algae were the most sensitive organisms not only for the combination products but mostly also for the individual a.s. present in these products. In contrast to the finding for the previously discussed herbicidal products, the toxicity of these individual fungicidal a.s. was very high for the same organisms that were most sensitive for the products (i.e., algae). This means, that formulation additives would need to be highly toxic to algae to notably contribute to the overall mixture toxicity to algae. Taking all this evidence together, it appears unlikely that formulation additives were the reason for the underestimation of the algal toxicity of products containing fenpropidin or fenpropimorph. In agreement with this is the observation that the algal toxicity of the mono-formulation (expressed in content a.s.) and the technical a.s. differed by less than factor 3 for fenpropimorph (no information for such a comparison is available for fenpropidin). This leaves as possible explanation a synergistic interaction between some members of subgroups of SBI fungicides, particularly those from the subgroups G2 and G1, in algae. Yet, this evidence of synergism between the G1 and G2 subgroups of SBI fungicides is based on a database analysis only and would certainly need experimental verification and further elucidation of the modes of actions of these fungicides in algae. Likewise, the lack of evidence for synergism between the G2 fungicide spiroxamine and DMI fungicides remains to be explained by further investigations. Based on the extensive review of Belden et al. [15] and own literature research, neither fenpropidin, fenpropimorph, or spiroxamine together with DMI fungicides have so far been explicitly tested for their mixture toxicity.

\section{Implications for considering mixture toxicity in the regulatory context}

The higher frequency and larger degree of deviation between predicted and observed mixture toxicity found in the present study in comparison to previous results $[14,15]$ suggests that the rather high degree of reliability of mixture toxicity predictions established so far in the scientific literature cannot be directly transferred to the regulatory context. A considerable part of the uncertainty in the prediction was caused by the much larger heterogeneity of the data used as input for the prediction. These data do not fulfill in all aspects the pre-conditions of scientific mixture toxicity concepts but are characterized by a considerable degree of data heterogeneity due to variation among laboratories, test protocols, test species, and measured endpoints even when a considerable effort is made to select toxicity data that were derived in similar tests (first data set). Importantly, the data heterogeneity did not lead to a bias, i.e., a systematic over-or underestimation of product toxicity, but only increased the uncertainty of the mixture toxicity prediction.

The influence of formulation additives was shown here to be a presumably highly relevant factor that reduces the reliability of mixture toxicity predictions that are based solely on the a.s. in a product. Information on additives as foreseen by the new regulation on placing PPP on the market [2] is expected to reduce this source of uncertainty in the future as it may allow including genuine toxicity of the additives into the prediction. Particularly the option to "bridge" toxicity estimates across PPP with similar or even identical a.s. 
composition but different formulation additives may be improved by mixture toxicity predictions coupled with toxicity information on additives.

The reliability of the mixture toxicity prediction is a key aspect when aiming at establishing a predictive mixture toxicity assessment in the regulatory context, e.g., in the environmental risk assessment of PPP. Any regulatory decision based on predicted mixture toxicity instead of measured product toxicity needs to take into account eventual additional uncertainty related to this prediction. Particularly the risk of underestimating the actual mixture toxicity should be minimized, while overprotective decisions resulting from overestimation of mixture toxicity by the prediction could be revised by conducting actual ecotoxicological tests with the combination product (which represents the whole-mixture testing approach). In this context, the current impossibility to reliably predict chronic mixture toxicity for Daphnia and fish is particularly disadvantageous. For these endpoints, product toxicity data are rarely available to risk assessors and more frequent product testing is undesirable in view of animal welfare. Furthermore, component-based predictions may be particularly desirable for chronic toxicity because the composition of the environmentally relevant mixture to which long-term exposure occurs may considerably differ from the original product composition due to, e.g., different environmental fate of the mixture components following application, rendering product test data eventually not very relevant. A possible solution in the future would be to report $\mathrm{EC}_{x}\left(\mathrm{EC}_{10}\right.$ or $\left.\mathrm{EC}_{20}\right)$ values in chronic Daphnia and fish studies with the a.s. However, this approach would require different test designs and, if $\mathrm{EC}_{x}$ values are to be derived simply in addition to NOEC values in mixed NOEC/EC $C_{x}$ designs, also the use of more concentrations levels and, hence, more animals. No extrapolation of the here presented results to higher-tier studies such as aquatic mesocosm studies appears currently possible. How mixture toxicity can be considered in the higher-tier risk assessment remains therefore an open question beyond the scope of the present study.

When combining toxicity data derived in tests as similar as possible (as exemplified with the first data set), a considerably frequent underestimation of product toxicity was found with underestimations by more than factor 100 in $0-13 \%$ of cases, depending on the endpoint. Even the more conservative approach of combining the lowest toxicity values within each of the six endpoints for the mixture toxicity predictions (as exemplified with the second data set) resulted in a more than 100-fold underestimation of product toxicity in $0 \%$ to up to $9 \%$ of cases. As shown for some herbicidal combination products, the degree of these underestimations could become relevant in the regulatory context.
A twofold deviation has been applied as a threshold to denote compliance between predicted and observed mixture toxicity in the present study, in accordance with previous studies $[14,15]$. A greater deviation (e.g., up to factor 5 or even factor 10) may be seen as still not indicating non-additive interactions given the impact of the large heterogeneity in the input data base. Establishing a safety factor may be an option to take into account the uncertainty in the mixture toxicity prediction. The present study indicates that with a safety factor of 100 applied to a mixture-toxicity prediction, up to about $10 \%$ of "wrong" decisions have to be anticipated in some endpoints for some types of PPP. Based on the present study, such a safety factor could be reduced on a caseby-case basis, depending on the assessed endpoint and pesticide combination. When applying the more conservative approach of using the lowest toxicity measures within each endpoint for the prediction, a safety factor of 5 for the acute toxicity ( $E C_{x}$-based) of fungicidal products to Daphnia and fish as well as a safety factor of 10 for the toxicity of herbicidal products to primary producers would result in less than 5\% of "wrong" decisions. Yet, it is important to note that such "wrong" decisions would only result in non-protective decisions if the respective endpoint is indeed driving the risk assessment for the aquatic compartment.

\section{Conclusions}

The here investigated commercial plant protection products represent mixtures of pesticides intentionally released into the environment and are therefore highly representative for mixtures of pesticides occurring in the environment. The database extensively covers fungicides and herbicides, but does not allow extrapolations to other types of pesticides such as mixtures with insecticides. Furthermore, the present study covered a larger diversity of different combinations of pesticidal modes of action than previous investigations and included the so far underrepresented group of fungicides. The finding of few but large deviations between mixture toxicity prediction and observation for these commercial pesticide mixtures warns against simply extrapolating conclusions on uncertainty derived from data obtained in welldesigned scientific studies to the regulatory risk assessment context as this may easily overestimate the reliability of decisions. This holds, to a lesser degree, also for the more conservative approach of using the lowest toxicity measures for each a.s. within a specified endpoint as input data for the mixture toxicity predictions instead of the (scientifically more correct) toxicity measures derived with the same test organisms in similar tests. The reliability of the CA prediction depended on the type of pesticide combination and varied for the same combination among different taxonomic groups. 
Some evidence for a synergistic interaction was found for a combination of fungicides that has so far not been systematically tested for joint algal toxicity, namely the G2-fungicides fenpropidin and fenpropimorph in combination with DMI fungicides. Apart from this specific case, deviations between prediction and observation appeared to be due to the heterogeneity of input data or caused by the influence of the formulation additives that have been disregarded in the mixture toxicity predictions. Formulation additives are suspected to be the reason for unexpected high product toxicity particularly in the case of products where the tested organisms are rather insensitive for the a.s contained in the product such as for example fish in the case of specifically acting herbicides. For some clearly defined endpoints, the compliance between prediction and observation was found to be unbiased and sufficiently reliable to base regulatory decisions for combination products on CA predictions, eventually applying an additional safety factor of up to 10. These endpoints were acute Daphnia and fish toxicity in the case of fungicidal combination products as well as growth inhibition of aquatic primary producers in the case of herbicidal combination products.

\section{Methods}

\section{Combination products}

Plant protection products with two or more a.s. (i.e., combination products) registered in Germany (as of August 2008) were analyzed with regard to their composition using the database of the German Federal Office of Consumer Protection and Food Safety. Data on the aquatic toxicity of the registered herbicidal and fungicidal combination products were compiled from the database of the Federal Environment Agency (UBA), Germany, and reflect the information submitted by applicants for the national registration of these products. Information on the a.s. composition and aquatic toxicity of combination products registered in Germany is publicly available through the competent authorities (http:// www.bvl.bund.de).

The single-species aquatic toxicity endpoints of the combination products assessed in the present study were algal growth inhibition measured as growth rate or biomass $\left(\mathrm{EC}_{50} ; 3,4\right.$, or 5 days of static exposure; mostly the green algae Pseudokirchneriella subcapitata and Desmodesmus subspicatus), Lemna growth inhibition $\left(\mathrm{EC}_{50}\right.$; 7 or 14 days of static or semi-static exposure; mostly Lemna gibba), immobility of aquatic invertebrates (acute toxicity, $\mathrm{EC}_{50} ; 48 \mathrm{~h}$ of static, semi-static, or flow-through exposure; always Daphnia magna), mortality of fish (acute toxicity, $\mathrm{LC}_{50} ; 96 \mathrm{~h}$ of static, semi-static, or flowthrough exposure; mostly Oncorhynchus mykiss), inhibition of reproduction of aquatic invertebrates (chronic toxicity, NOEC; 21 days of semi-static or flow-through exposure; always D. magna), and chronic toxicity to fish (NOEC; static, semi-static, or flow-through exposure with various test methods and biological endpoints; mostly O. mykiss). Used toxicity measures for the products are based on nominal concentrations, because only in rare cases the concentration of all a.s. contained in the product had been confirmed by analytical chemistry.

\section{Active substances}

As for the products, the toxicity measures for the individual a.s. relate mostly to nominal concentrations. In many of these studies, the test concentrations of the a.s. were confirmed by analytical chemistry as falling between $80-120 \%$ of the nominal concentration, which is in test guidelines usually defined as the acceptable range allowing the further use of nominal concentrations. Potential differences between nominal and actual test concentration, regardless if measured or not, are considered in the present study as contributing to data heterogeneity. Only effect concentrations obtained with the technical a.s. were used in the present study in order to avoid interference with formulation additives when using data derived with formulated a.s.

The first data set ("most similar endpoints") was derived from the database of the Federal Environment Agency (UBA), Germany, which includes all aquatic toxicity studies submitted by different applicants in the course of PPP authorization in Germany since about more than one decade. Within each of the six aquatic endpoints described above for the products, the a.s. tests that were most similar to the test with the respective product were selected from the extensive number of tests with different species, exposure conditions, and measured toxic effects available in the data base. If, for example, the $\mathrm{EC}_{50}$ value of a combination product for the endpoint "algal growth inhibition" was derived in a test with $D$. subspicatus and based on biomass measurement after 3 days of static exposure, $\mathrm{EC}_{50}$ value for the a.s. present in the product was selected from a test that used (in the order of importance for the selection) the same species (D. subspicatus), the same measured toxic effect (biomass), the same exposure time (3 days) and the same exposure condition (static).

The second data set ("most sensitive endpoints") was derived from the list of endpoints identified during the European review process for new and existing a.s. [1]. The EU list of endpoints for each a.s. included in Annex I is publicly available. Data were obtained for the present study in December 2009 (http://ec.europa.eu/sanco_pesticides/public/index.cfm). With some rare exceptions, the toxicity data compiled from the EU list of endpoints represent for each a.s. the most sensitive species within a taxonomic group, i.e., the lowest 
toxicity measure for each of the six here investigated endpoints as selected by the EU review procedure from all available validated data. Compared to the data set "most similar endpoints", the heterogeneity in the data set "most sensitive endpoints" was much larger as more different species were combined to predict and compare mixture toxicity. For the endpoint algal growth inhibition, for example, diatoms and blue-green algae were also used in the prediction and compared to a value for the product that had been obtained with green algae, for example. Likewise, more different species were included in the second data set such as mysid shrimps (therefore, the endpoint was named acute toxicity to aquatic invertebrates in the second data set), other water plants than Lemna (growth inhibition of water plants) and various fish species. In addition, more diverse study types and effect measures are represented in the second data set as well as NOECs also derived from fish full life cycle tests, which never occurred in the data set of "most similar endpoints".

\section{Mode of action classification}

All a.s. in the investigated combination products were classified based on their pesticidal mode of action according to established classification systems $[16,17,36]$. Pesticidal modes of action were considered at the biochemical level, i.e., the targeted enzymes, using the respective subgroups such as for example G1 and G2 for inhibitors of ergosterol biosynthesis because the inhibition is realized through inhibition of different enzymes. Only the subgroups of photosystem II inhibitors (HRAC group C) were pooled into one group because they are assumed to act similarly by affecting closely related targets, e.g., different bindings sites at the same molecule $[9,32]$. While this classification scheme, being at least partly based on known molecular targets, could with some reason be called a mechanism-ofaction classification [37], we used the more general term mode-of-action also when more detailed mechanistic information is available about the underlying biochemical processes.

\section{Prediction of mixture toxicity and comparison with the toxicity of combination products}

The toxicity of a combination product with $n$ active substances was calculated according to CA using the equation given in Figure 1. It is important to notice that any contribution of the additives in the combination products was disregarded in the prediction, i.e., it was assumed that the toxicity of the commercial mixture solely depends on the toxicity of the a.s. In the case of chronic toxicity towards aquatic invertebrates and fish, NOEC values were used instead of $E_{50}$ values as the latter are usually not reported from such tests. This use of NOEC values (conducted here as exemplification) is a violation of the basic scientific concept of CA, because it assumes implicitly (and wrongly) that NOEC values (in analogy to $\mathrm{EC}_{\mathrm{x}}$ values) relate to a defined effect level, while in fact biological effects occurring at the NOEC differ among independent tests and depend strongly on the experimental design [13]. Only CA-based predictions were calculated in the present study because the available data pool did not allow for an application of IA.

The deviation between the toxicity predicted by CA and the observed toxicity of the combination product was quantified by calculating the model deviation ratio (MDR) that had been introduced by Belden et al. [15]. As illustrated in Figure 1, the MDR is the quotient of the predicted $\mathrm{EC}_{50}$ value for the mixture $\left(\mathrm{EC}_{50}\right.$ pred $)$ and the observed $\mathrm{EC}_{50}$ of the product $\left(\mathrm{EC}_{50}\right.$ obs $)$. Essential for this comparison is that both effect concentrations relate to the sum of a.s. in the mixture and the product, respectively. As $\mathrm{EC}_{50}$ values for products are often given related to the concentration of the product in the test medium, the concentration of the sum of a.s. for the product was re-calculated from the a.s. content of the product and its density, where applicable.

Censored data, i.e., a NOEC or $\mathrm{EC}_{50}$ value greater or smaller than a given value, resulted for example from limit tests (only one concentration is tested), but can also be obtained when testing several concentrations. In the case of censored $\mathrm{EC}_{50}$ or NOEC values, the "greater than" and "smaller than" information was kept in the calculation, resulting in a censored MDR value or, if for the product and at least one a.s. censored input data were given, in the MDR value not being determined.

\section{Statistics}

The frequency distributions of the calculated numerical MDR values are displayed based on nine categories of deviation using $0.01,0.1,0.2,0.5,2,5,10$, and 100 as borders of the group categories. Deviation by less than factor 2 (MDR between 0.5 and 2) was assumed as indicating compliance between prediction and observation $[14,15]$. Differences between groups (herbicidal and fungicidal products; endpoints) were analyzed by comparing the median MDR of the respective groups using the non-parametric Mood's median test (sign scores test). Spearman rank correlation was used to analyze explanatory relationships. All statistical analyses were conducted in Statistica, version 9.0.

\section{Acknowledgements}

This study was financially supported by the Federal Environment Agency (UBA), Germany, through projects FKZ 36003041 and FKZ 36003046 . The views expressed herein by the authors are their own and do not necessarily reflect the views or policies of governmental agencies. We thank Thomas Knacker for fruitful discussions and Rolf Altenburger for valuable comments on an earlier version of the manuscript. 


\section{Author details}

${ }^{1}$ ECT Oekotoxikologie GmbH, Boettgerstr. 2-14, 65439 Flörsheim, Germany ${ }^{2}$ Federal Environment Agency (UBA), Woerlitzer Platz 1, 06844 Dessau, Germany

\section{Authors' contributions}

Both authors collected the data. TF conceived of the study. AC conducted the analysis and drafted the manuscript. Both authors read and approved the final manuscript.

\section{Competing interests}

The authors declare that they have no competing interests.

Received: 17 March 2011 Accepted: 16 June 2011

Published: 16 June 2011

\section{References}

1. EC (European Council): Council Directive of 15 July 1991 concerning the placing of plant protection products on the market. 2004, 91/414/EEC

2. EC (European Council): Regulation of 21 October 2009 concerning the placing of plant protection products on the market and repealing Council Directives 79/117/EEC and 91/414/EEC. 2009, EC No 1107/2009.

3. Weinhold B: Mystery in a bottle: will the EPA require public disclosure of inert pesticide ingredients? Environ Health Perspect 2010, 118:A168-171.

4. Junghans M, Backhaus T, Faust M, Scholze M, Grimme LH: Application and validation of approaches for the predictive hazard assessment of realistic pesticide mixtures. Aquat Toxicol 2006, 76:93-110.

5. Gilliom RJ: Pesticides in U.S. streams and groundwater. Environ Sci Technol 2007, 41:3409-3414.

6. Verro R, Finizio A, Otto $S$, Vighi M: Predicting pesticide environmental risk in intensive agricultural areas. II: Screening level risk assessment of complex mixtures in surface waters. Environ Sci Technol 2009, 43:530-537.

7. Calamari D, Vighi M: A proposal to define quality objectives for aquatic life for mixtures of chemical substances. Chemosphere 1992, 25:531-542.

8. De Zwart D, Posthuma L: Complex mixture toxicity for single and multiple species: proposed methodologies. Environ Toxicol Chem 2005, 24:2665-2676.

9. Chèvre N, Loepfe C, Singer H, Stamm C, Fenner K, Escher BI: Including mixtures in the determination of water quality criteria for herbicides in surface water. Environ Sci Technol 2006, 40:426-435.

10. Altenburger $\mathrm{R}$, Walter $\mathrm{H}$, Grote $\mathrm{M}$ : What contributes to the combined effect of a complex mixture? Environ Sci Technol 2004, 38:6353-6362.

11. Backhaus T, Arrhenius A, Blanck H: Toxicity of a mixture of dissimilarly acting substances to natural algal communities: predictive power and limitations of independent action and concentration addition. Environ Sci Technol 2004, 38:6363-6370.

12. Olmstead AW, LeBlanc GA: Toxicity assessment of environmentally relevant pollutant mixtures using a heuristic model. Integr Environ Assess Manag 2005, 1:114-122.

13. Kortenkamp A, Backhaus T, Faust M: State of the art report on mixture toxicity 2009, Final Report to the European Commission under Contract Number 070307/2007/485103/ETU/D.1..

14. Deneer JW: Toxicity of mixtures of pesticides in aquatic systems. Pest Manag Sci 2000, 56:516-520.

15. Belden JB, Gilliom RJ, Lydy MJ: How well can we predict the toxicity of pesticides mixtures to aquatic life? Integr Environ Assess Manag 2007, 3:364-372.

16. Herbicide Resistance Action Committee, HRAC: The world of herbicidesHRAC classification on mode of action 2000. 2008 [http://www. plantprotection.org/hrac]

17. Fungicide Resistance Action Committee, FRAC: Mode of action of fungicides-FRAC classification on mode of action 2007. 2008 [http:// www.frac.info]

18. De Laender F, Janssen CR, De Schamphelaere KAC: Non-simultaneous ecotoxicity testing of single chemicals and their mixture results in erroneous conclusions about the joint action of the mixture. Chemosphere 2009, 76:428-432.

19. Crane M, Newman MC: What level of effect is a no observed effect? Environ Toxicol Chem 2000, 19:516-519.

20. Bliss Cl: The toxicity of poisons applied jointly. Ann Appl Biol 1939, 26:585-615.
21. Altenburger R, Boedeker W, Faust M, Grimme LH: Regulations for combined effects of pollutants: consequences from risk assessment in aquatic toxicology. Food Chem Toxicol 1996, 34:1155-1157.

22. Groten JP: Mixtures and interactions. Food Chem Toxicol 2000, 38:S65-S71

23. Borgert CJ, Quill TF, McCarthy LS, Mason AM: Can mode of action predict mixture toxicity for risk assessment? Toxicol Appl Pharmacol 2004, 201:85-96.

24. Berenbaum MC: What is synergy? Pharmacol Rev 1989, 41:93-141.

25. McCarthy LS, Borgert CJ: Review of the toxicity of chemical mixtures: Theory, policy, and regulatory practice. Regul Toxicol Pharmacol 2006, 45:119-143.

26. Cedergreen N, Christensen AM, Kamper A, Kudsk P, Mathiassen SK, Streibig J, Sorensen H: A review of independent action compared to concentration addition as reference models for mixtures of compounds with different molecular target sites. Environ Toxicol Chem 2008, 27:1621-1632.

27. Hassold E, Backhaus T: Chronic toxicity of five structurally diverse demethylase-inhibiting fungicides to the crustacean Daphnia magna: a comparative assessment. Environ Toxicol Chem 2009, 28:1218-1226.

28. Ankley GT, Jensen KM, Durham EJ, Makynen EA, Butterworth BC, Kahl MD, Villeneuve DL, Linnum A, Gray LE, Cardon M, Wilson VS: Effects of two fungicides with multiple modes of action on reproductive endocrine functions in the fathead minnow (Pimephales promelas). Toxicol Sci 2005, 86:300-308.

29. Stenersen J: Chemical pesticides: mode of action and toxicology Boca Raton, FL, USA: CRC Press; 2004

30. Junghans M, Backhaus T, Faust M, Scholze M: Predictability of combined effects of eight chloroacetanilide herbicides on algal reproduction. Pest Manag Sci 2003, 59:1101-1110.

31. Backhaus T, Faust M, Scholze M, Gramatica P, Vighi M, Grimme LH: Joint algal toxicity of phenylurea herbicides is equally predictable by concentration addition and independent action. Environ Toxicol Chem 2004, 23:258-264

32. Faust $M$, Altenburger $R$, Boedeker W, Grimme LH: Additive effects of herbicide combinations on aquatic non-target organisms. Sci Total Environ 1993, Suppl. Part 2: 941-952.

33. Standing Committee on the Food Chain and Animal Health, SC: Review report for the active substance iodosulfuron. 2003, SANCO/10166/2003Final.

34. Cox C, Surgan M: Unidentified inert substances in pesticides: Implications for human and environmental health. Environ Health Perspect 2006, 114:1803-1806.

35. Hazen JL: Adjuvants-Terminology, classification, and chemistry. Weed Technol 2000, 14:773-784.

36. Tomlin CDS: The e-Pesticide Manual. 14 edition. British Crop Protection Council; 2008

37. Escher $\mathrm{Bl}$, Hermens JL: Modes of action in ecotoxicology: their role in body burdens, species sensitivity, QSARs, and mixture effects. Environ Sci Technol 2002, 36(20):4201-4217.

doi:10.1186/2190-4715-23-22

Cite this article as: Coors and Frische: Predicting the aquatic toxicity of commercial pesticide mixtures. Environmental Sciences Europe 2011 23:22.

\section{Submit your manuscript to a SpringerOpen ${ }^{\circ}$ journal and benefit from:}

- Convenient online submission

- Rigorous peer review

- Immediate publication on acceptance

- Open access: articles freely available online

- High visibility within the field

- Retaining the copyright to your article

Submit your next manuscript at $>$ springeropen.com 